\title{
Switched Singular Linear Systems
}

\author{
Josep Clotet \\ Dep. Matemàtica Aplicada I \\ Universitat Politècnica de Catalunya \\ 08028 Barcelona - Spain \\ Email: josep.clotet@upc.edu
}

\author{
Josep Ferrer \\ Dep. Matemàtica Aplicada I \\ Universitat Politècnica de Catalunya \\ 08028 Barcelona - Spain \\ Email: josep.ferrer@upc.edu
}

\author{
M. Dolors Magret \\ Dep. Matemàtica Aplicada I \\ Universitat Politècnica de Catalunya \\ 08028 Barcelona - Spain \\ Email: m.dolors.magret@upc.edu
}

\begin{abstract}
We consider switched singular linear systems and determine the set of reachable/controllable states. We derive necessary and sufficient conditions for such a system to be reachable/controllable when an "equisingularity condition" holds.
\end{abstract}

Index Terms-Switched linear system, singular system, controllability.

\section{INTRODUCTION}

At the end of the 1970s singular linear systems started to attract attention of researches due to the fact that they appear in different areas, for example when modeling complex social, economic, chemical and biological systems. Practical examples have been studied by Campbell and Rose, Haggman and Bryant, Luenberger, Petzold, Singh and Liu, Wang and Dai, among many other authors (see [2], [7], [9], [10], [11], [13]). Many of classical systems results have been generalized to the case of singular linear systems. A very complete survey of the new methods required to study singular systems (traditional approaches are not suitable for their study) is [4].

On the other hand, switched linear systems have been widely studied, specially since the 1990s. They naturally arise from physics and can be found in various fields such as electrical and electronic engineering (generation of electric power, as well as electrical devices such as motors and transformers), aeronautical or automotive. See, for example, the works by Brockett and Wood, Corona, Giua and Seatzu, Feuer, Goodwin and Salgado, Ge, Hang, Lee and Zhang (see [1], [3], [5], [6]). Roughly speaking, a switched system is a family of continuous-time (or discrete-time) dynamical subsystems and a rule that determines the switching between them. [12] is a nice and complete survey of this topic.

The importance of both types of systems, suggested to attempt a further step towards the study of switching singular systems.

In this paper we focus on obtaining the set of reachable/controllable states. The main contribution of the paper is a characterization of reachable/controllable systems when an "equisingularity condition" holds.

In $\S 2$, we define switched singular linear systems.

In $\S 3$, we present a simple example whose description yields such a system.

In $\S 4$, we obtain the set of reachable/controllable states and derive a characterization of reachable/controllable systems.

Finally, $\S 5$ is devoted to the conclusions. mds

January 16, 2009

\section{Switched Singular LineAR Systems}

A switched system is a system which consists of several subsystems and a rule that orchestrates the switching between them.

We introduce here the concept of switched singular linear system, that is to say, a switching system consisting of several singular linear subsystems. Definitions in this Section are analogous to those in [12] for switched non-singular systems.

Definition 1: A switched singular linear system is a system which consists of several linear singular subsystems and a piecewise constant map $\sigma$ taking values into the index set $M=\{1, \ldots, m\}$ which indexes the different subsystems. In the continuous case, such a system can be mathematically described by

$$
\left\{\begin{aligned}
E_{\sigma} \dot{\mathbf{x}}(t) & =A_{\sigma} \mathbf{x}(t)+B_{\sigma} u(t) \\
\mathbf{y}(t) & =C_{\sigma} \mathbf{x}(t)
\end{aligned}\right.
$$

where $E_{\sigma}, A_{\sigma} \in M_{n}(\mathbb{R}), B_{\sigma} \in M_{n \times m}(\mathbb{R}), C_{\sigma} \in M_{p \times n}(\mathbb{R})$, rk $E_{\sigma}<n$. In the discrete case an analogous description can be done.

Definition 2: Given an initial time $t_{0}$, a switching path is a function of time

$\theta:\left[t_{0}, T\right) \longrightarrow M, T>t_{0}$.

Definition 3: A switching path $\theta$ is said to be well-defined on $\left[t_{0}, T\right)$ if it is defined in $\left[t_{0}, T\right)$ and for all $t \in\left[t_{0}, T\right)$, both $\lim _{s \longrightarrow t^{+}} \theta(s)$ and $\lim _{s \longrightarrow t^{-}} \theta(s)$ exist and the set

$$
\left\{t \in\left[t_{0}, T\right) \mid \lim _{s \longrightarrow t^{+}} \theta(s) \neq \lim _{s \longrightarrow t^{-}} \theta(s)\right\}
$$

is finite for any finite sub-interval of $\left[t_{0}, T\right.$ ) (in the case where $t=t_{0}$, we will consider $\left.\lim _{s \longrightarrow t_{0}^{-}} \theta(s)=\theta\left(t_{0}\right)\right)$.

A well-defined switching path is uniquely determined by a switching sequence

$$
\left\{\left(\left[t_{0}, t_{1}\right), \sigma\left(t_{0}^{+}\right)\right), \ldots,\left(\left[t_{\ell}, t_{\ell+1}\right), \sigma\left(t_{\ell}^{+}\right)\right)\right\}
$$

being $\sigma(t)=\sigma\left(t_{i}^{+}\right)=\lim _{s \longrightarrow t_{i}^{+}} \sigma(s)$ if $t \in\left[t_{i}, t_{i+1}\right)$ for $0 \leq i \leq$ $\ell, t_{\ell+1}=T$.

We will restrict ourselves to the case of a continuous system with two subsystems and $\sigma=\sigma(t)$ the rule which decides the change between them. 
The case where more than two subsystems are considered or the discrete case can be handled analogously.

\section{A Simple EXAmple}

Let us consider a PWM (pulse-width modulator) boostconverter. This converter is used in many applications, as a dc-dc converter, to obtain a voltage higher than the battery source one. Over the last two decades, several approaches to modeling them have been proposed.

The boost converter is an example of a very simple switched linear system. See, for example, [8]. The switch is controlled with a pulse-width modulator. For any position of the switch, the corresponding system is linear. There are obviously two such systems. The state of the switch is completely determined by an input $\mathrm{s}(\mathrm{t})$.

We will (as usual) denote by $L$ an inductance, $C$ a capacitance, $R$ a load resistance and $e_{S}(t)$ the source voltage. The switch has state $s(t)$. With this converter it is possible to transform the source voltage $e_{S}(t)$ into a higher voltage $e_{C}(t)$ over the load $R$.

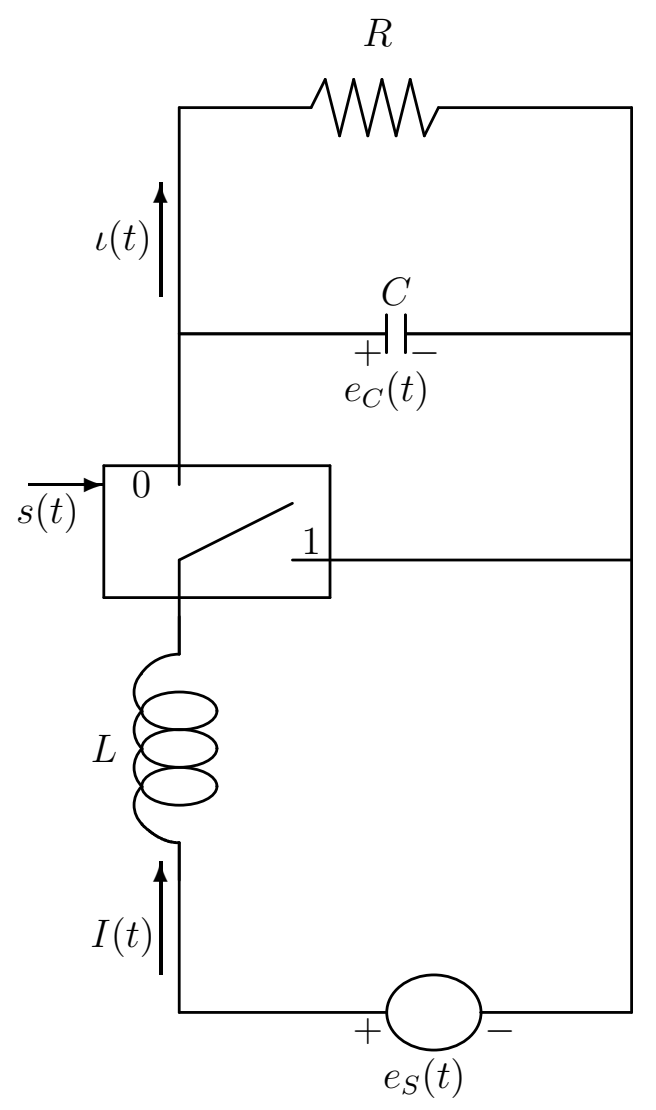

Fig. 1. The boost converter

To get a state space model, we need to select some variables. This selection is obviously not unique, therefore the model is not unique. Appropriate models are established, and the obtained description state equation is required to be convenient for system analysis and/or design. In our case, with a similar selection of functions to that in [4] as state variables:

$$
\mathbf{x}_{1}(t)=I(t) \quad \mathbf{x}_{2}(t)=e_{C}(t) \quad \mathbf{x}_{3}(t)=R \iota(t)-e_{R}(t)
$$

applying Kirchoff's laws, in matritial notation, the state equation is:

$$
\begin{aligned}
\left(\begin{array}{lll}
1 & 0 & 0 \\
0 & 1 & 0 \\
0 & 0 & 0
\end{array}\right)\left(\begin{array}{c}
\dot{\mathbf{x}}_{1}(t) \\
\dot{\mathbf{x}}_{2}(t) \\
\dot{\mathbf{x}}_{3}(t)
\end{array}\right) \\
=\left(\begin{array}{ccc}
0 & \frac{-1}{L} & 0 \\
\frac{1}{C} & \frac{-1}{R C} & 0 \\
0 & 0 & 1
\end{array}\right)\left(\begin{array}{l}
\mathbf{x}_{1}(t) \\
\mathbf{x}_{2}(t) \\
\mathbf{x}_{3}(t)
\end{array}\right)+\left(\begin{array}{c}
\frac{1}{L} \\
0 \\
0
\end{array}\right) e_{s}(t)
\end{aligned}
$$

if the switch is on $(s(t)=0)$, and

$$
\begin{aligned}
\left(\begin{array}{lll}
1 & 0 & 0 \\
0 & 1 & 0 \\
0 & 0 & 0
\end{array}\right)\left(\begin{array}{l}
\dot{\mathbf{x}}_{1}(t) \\
\dot{\mathbf{x}}_{2}(t) \\
\dot{\mathbf{x}}_{3}(t)
\end{array}\right) \\
=\left(\begin{array}{ccc}
0 & 0 & 0 \\
0 & -\frac{1}{R C} & 0 \\
0 & 0 & 1
\end{array}\right)\left(\begin{array}{l}
\mathbf{x}_{1}(t) \\
\mathbf{x}_{2}(t) \\
\mathbf{x}_{3}(t)
\end{array}\right)+\left(\begin{array}{c}
\frac{1}{L} \\
0 \\
0
\end{array}\right) e_{s}(t)
\end{aligned}
$$

if the switch is off $(s(t)=1)$.

\section{Reachable States. Controllability}

Let us consider a system $\Sigma$ defined by the following linear subsystems:

$$
\begin{aligned}
\Sigma_{1}\left\{\begin{aligned}
E \dot{\mathbf{x}}(t) & =A \mathbf{x}(t)+B u(t) \\
\mathbf{y}(t) & =C \mathbf{x}(t)
\end{aligned}\right. \\
\Sigma_{2}\left\{\begin{aligned}
E^{\prime} \dot{\mathbf{x}}(t) & =A^{\prime} \mathbf{x}(t)+B^{\prime} u(t) \\
\mathbf{y}(t) & =C^{\prime} \mathbf{x}(t)
\end{aligned}\right.
\end{aligned}
$$

where $E, E^{\prime}, A, A^{\prime} \in M_{n}(\mathbb{R}), B, B^{\prime} \in M_{n \times m}(\mathbb{R}), C, C^{\prime} \in$ $M_{p \times n}(\mathbb{R})$, with rk $E$, rk $E^{\prime}<n$, and a rule $\sigma$ which defines the switching between them.

We will consider the case where the matrix pencils $\lambda E+$ $A, \lambda E^{\prime}+A^{\prime}$ are regular, as it is usual, in order to ensure that the systems have a unique solution for any sufficiently differentiable function $u(t)$. Under this regularity assumption, there exist invertible matrices $Q, Q^{\prime}, P, P^{\prime} \in G l_{n}(\mathbb{R})$ such that $Q E P=\operatorname{diag}\left(I_{n_{1}}, \mathcal{N}\right), Q^{\prime} E^{\prime} P^{\prime}=\operatorname{diag}\left(I_{n_{1}^{\prime}}, \mathcal{N}^{\prime}\right), Q A P=$ $\operatorname{diag}\left(A_{1}, I_{n_{2}}\right)$ and $Q^{\prime} A^{\prime} P^{\prime}=\operatorname{diag}\left(A^{\prime}{ }_{1}, I_{n^{\prime}}{ }_{2}\right)$, with $n_{1}+n_{2}=$ $n^{\prime}{ }_{1}+n^{\prime}{ }_{2}=n$ and $\mathcal{N}, \mathcal{N}^{\prime}$ nilpotent matrices.

Let us denote by $\Phi\left(t, t_{0}, x_{0}, u, \sigma\right)$ the state trajectory (or solution of the system) at time $t$ of the continuous-time switched singular system starting from $x\left(t_{0}\right)=x_{0}$ with input $u$ and switching well-defined path determined by the switching sequence $\sigma$.

From now on, we will assume that the function $u(t)$ is a $h$ times piecewise continuous differentiable function ( $h$ the maximum of nilpotent indices of matrices $\mathcal{N}, \mathcal{N}^{\prime}$ ), and that for system $\Sigma$ the following (quite natural in applications such in the example above) "equisingularity condition" holds: there exists a state variables change which yields, jointly with convenient pre-multiplication of both state equations to make 
possible that the equations of the subsystems could be written in the form:

$$
\begin{aligned}
& \Sigma_{1}\left\{\begin{array}{c}
\left(\begin{array}{cc}
I_{n_{1}} & 0 \\
0 & \mathcal{N}
\end{array}\right)\left(\begin{array}{c}
\dot{\mathbf{x}}^{1}(t) \\
\dot{\mathbf{x}}^{2}(t)
\end{array}\right) \\
=\left(\begin{array}{cc}
A_{1} & 0 \\
0 & I_{n_{2}}
\end{array}\right)\left(\begin{array}{l}
\mathbf{x}^{1}(t) \\
\mathbf{x}^{2}(t)
\end{array}\right)+\left(\begin{array}{c}
B_{1} \\
B_{2}
\end{array}\right) u(t) \\
\mathbf{y}(t)=\left(\begin{array}{ll}
C_{1} & C_{2}
\end{array}\right)\left(\begin{array}{l}
\mathbf{x}^{1}(t) \\
\mathbf{x}^{2}(t)
\end{array}\right)
\end{array}\right. \\
& \Sigma_{2}\left\{\begin{array}{c}
\left(\begin{array}{cc}
I_{n_{1}} & 0 \\
0 & \mathcal{N}^{\prime}
\end{array}\right)\left(\begin{array}{c}
\dot{\mathbf{x}}^{1}(t) \\
\dot{\mathbf{x}}^{2}(t)
\end{array}\right) \\
=\left(\begin{array}{cc}
A^{\prime}{ }_{1} & 0 \\
0 & I_{n_{2}}
\end{array}\right)\left(\begin{array}{l}
\mathbf{x}^{1}(t) \\
\mathbf{x}^{2}(t)
\end{array}\right)+\left(\begin{array}{c}
B^{\prime}{ }_{1} \\
B^{\prime}{ }_{2}
\end{array}\right) u(t) \\
\mathbf{y}(t)=\left(\begin{array}{ll}
C^{\prime}{ }_{1} & C^{\prime}{ }_{2}
\end{array}\right)\left(\begin{array}{l}
\mathbf{x}^{1}(t) \\
\mathbf{x}^{2}(t)
\end{array}\right)
\end{array}\right.
\end{aligned}
$$

giving rise to the standard decomposition:

$$
\begin{aligned}
& \Sigma_{1}\left\{\begin{array}{c}
\Sigma_{1}^{l}\left\{\begin{array}{c}
\dot{\mathbf{x}}^{1}(t)=A_{1} \mathbf{x}^{1}(t)+B_{1} u(t) \\
\mathbf{y}^{1}(t)=C_{1} \mathbf{x}^{1}(t)
\end{array}\right. \\
\Sigma_{1}^{f}\left\{\begin{array}{c}
\mathcal{N} \dot{\mathbf{x}}^{2}(t)=\mathbf{x}^{2}(t)+B_{2} u(t) \\
\mathbf{y}^{2}(t)=C_{2} \mathbf{x}^{2}(t) \\
\mathbf{y}(t)=\mathbf{y}^{1}(t)+\mathbf{y}^{2}(t)
\end{array}\right.
\end{array}\right. \\
& \Sigma_{2}\left\{\begin{array}{c}
\Sigma_{2}^{l}\left\{\begin{array}{c}
\dot{\mathbf{x}}^{1}(t)=A^{\prime}{ }_{1} \mathbf{x}^{1}(t)+B^{\prime}{ }_{1} u(t) \\
\mathbf{y}^{1}(t)=C^{\prime}{ }_{1} \mathbf{x}^{1}(t)
\end{array}\right. \\
\Sigma_{2}^{f}\left\{\begin{array}{c}
\mathcal{N}^{\prime} \dot{\mathbf{x}}^{2}(t)=\mathbf{x}^{2}(t)+B^{\prime}{ }_{2} u(t) \\
\mathbf{y}^{2}(t)=C^{\prime}{ }_{2} \mathbf{x}^{2}(t) \\
\mathbf{y}(t)=\mathbf{y}^{1}(t)+\mathbf{y}^{2}(t)
\end{array}\right.
\end{array}\right.
\end{aligned}
$$

$\Sigma_{1}^{l}, \Sigma_{2}^{l}$ are called slow subsystems and $\Sigma_{1}^{f}, \Sigma_{2}^{f}$ are called fast subsystems $\left(\mathbf{x}^{1}\right.$ and $\mathbf{x}^{2}$ are the slow and fast sub-states, respectively).

That is to say, we assume that we can obtain this reduced form through invertible matrices $P=P^{\prime}, Q$ and $Q^{\prime}$ and that $n_{1}=n^{\prime}{ }_{1}$ (therefore $n_{2}=n^{\prime}{ }_{2}$ ).

We introduce the following definitions, which are the natural generalizations of those in the case of switched non-singular systems and in the case of singular systems (see [12] and [4]).

Definition 4: A state $x_{f}=\left(x_{f}^{1}, x_{f}^{2}\right) \in \mathbb{R}^{n_{1}} \times \mathbb{R}^{n_{2}}$ is reachable from 0 if there exists $t_{f}>t_{0}$, a switching welldefined path $\theta:\left[t_{0}, t_{f}\right] \longrightarrow M=\{1,2\}$ determined by a switching sequence $\sigma:\left[t_{0}, t_{f}\right) \longrightarrow\{1,2\}$ (we will consider $\left.\theta\left(t_{f}\right)=\theta\left(t_{f}^{-}\right)=\lim _{s \longrightarrow t_{f}^{-}} \theta(s)\right)$ and a control $u:\left[t_{0}, t_{f}\right] \longrightarrow$ $\mathbb{R}^{m}$ such that:
1. $x_{f}^{1}=\Phi\left(t_{f}, t_{0}, 0, u, \sigma\right)$; that is to say, $x_{f}^{1}$ is a reachable state by the switching system composed by the slow subsystems:

$$
\begin{aligned}
& \Sigma_{1}^{l}\left\{\begin{aligned}
\dot{\mathbf{x}}^{1}(t) & =A_{1} \mathbf{x}^{1}(t)+B_{1} u(t) \\
\mathbf{y}^{1}(t) & =C_{1} \mathbf{x}^{1}(t)
\end{aligned}\right. \\
& \Sigma_{2}^{l}\left\{\begin{array}{l}
\dot{\mathbf{x}}^{1}(t)=A^{\prime}{ }_{1} \mathbf{x}^{1}(t)+B^{\prime}{ }_{1} u(t) \\
\mathbf{y}^{1}(t)=C^{\prime}{ }_{1} \mathbf{x}^{1}(t)
\end{array}\right.
\end{aligned}
$$

2. $x_{f}^{2}=-\sum_{i=0}^{h-1} \mathcal{N}^{i} B_{2} u^{(i)}\left(t_{f}\right)$ in the case where $\sigma\left(t_{f}^{-}\right)=$ $\lim _{s \rightarrow t_{f}^{-}} \sigma(s)=1$,

$x_{f}^{2}=-\sum_{i=0}^{h-1} \mathcal{N}^{\prime i} B^{\prime}{ }_{2} u^{(i)}\left(t_{f}\right)$ in the case where $\sigma\left(t_{f}^{-}\right)=$ $\lim _{s \rightarrow t_{f}^{-}} \sigma(s)=2$.

Definition 5: A state $x_{f}=\left(x_{f}^{1}, x_{f}^{2}\right) \in \mathbb{R}^{n_{1}} \times \mathbb{R}^{n_{2}}$ is controllable if there exists $t_{f}>t_{0}$, a switching well-defined path $\theta:\left[t_{0}, t_{f}\right] \longrightarrow M=\{1,2\}$ determined by a switching sequence $\sigma:\left[t_{0}, t_{f}\right) \longrightarrow\{1,2\}$ (we consider $\theta\left(t_{f}\right)=\theta\left(t_{f}^{-}\right)=$ $\lim \theta(s))$ and a control $u:\left[t_{0}, t_{f}\right] \longrightarrow \mathbb{R}^{m}$ such that:

1'. $\Phi\left(t_{f}, t_{0}, x_{f}^{1}, u, \sigma\right)=0$, that is to say, $x_{f}^{1}$ is a controllable state by the switching system composed by the slow subsystems:

$$
\begin{aligned}
& \Sigma_{1}^{l}\left\{\begin{aligned}
\dot{\mathbf{x}}^{1}(t) & =A_{1} \mathbf{x}^{1}(t)+B_{1} u(t) \\
\mathbf{y}^{1}(t) & =C_{1} \mathbf{x}^{1}(t)
\end{aligned}\right. \\
& \Sigma_{2}^{l}\left\{\begin{array}{l}
\dot{\mathbf{x}}^{1}(t)=A^{\prime}{ }_{1} \mathbf{x}^{1}(t)+B^{\prime}{ }_{1} u(t) \\
\mathbf{y}^{1}(t)=C^{\prime}{ }_{1} \mathbf{x}^{1}(t)
\end{array}\right.
\end{aligned}
$$

2'. $x_{f}^{2}=-\sum_{i=0}^{h-1} \mathcal{N}^{i} B_{2} u^{(i)}\left(t_{f}\right)$ if $\sigma\left(t_{f}^{-}\right)=\lim _{s \longrightarrow t_{f}^{-}} \sigma(s)=1$, $x_{f}^{2}=-\sum_{i=0}^{h-1} \mathcal{N}^{\prime i} B^{\prime}{ }_{2} u^{(i)}\left(t_{f}\right)$ if $\sigma\left(t_{f}^{-}\right)=\lim _{s \longrightarrow t_{f}^{-}} \sigma(s)=$ 2.

In the following we will denote by $\langle M \mid N\rangle$ the vector subspace

$$
\operatorname{Im}\left[N, M N, M^{2} N, \ldots, M^{n-1} N\right]
$$

assuming that $M$ is a square matrix of order $n$ and $N \in$ $\mathcal{M}_{n \times m}(\mathbb{R})$.

Let us recall that both conditions, 1. and 1.' are equivalent (according to [12]) to:

$$
x_{f}^{1} \in \Re=\sum_{p=1}^{n_{1}} \Re_{p}
$$

where 


$$
\begin{aligned}
\Re_{1} & =\operatorname{Im}\left[B_{1}, B^{\prime}{ }_{1}\right] \\
\Re_{2} & =\Re_{1}+A_{1} \Re_{1}+A^{\prime}{ }_{1} \Re_{1}+\ldots+A_{1}^{n_{1}-1} \Re_{1}+A^{\prime n_{1}-1} \Re_{1} \\
& \ldots \\
\Re_{p+1} & =\Re_{p}+A_{1} \Re_{p}+A^{\prime}{ }_{1} \Re_{p}+\ldots+A_{1}^{n_{1}-1} \Re_{p}+A^{\prime n_{1}-1} \Re_{p} \\
& \ldots
\end{aligned}
$$

Note that there exists $\ell \leq n_{1}$ such that $\operatorname{dim} \Re_{\ell}=\operatorname{dim} \Re_{\ell+1}$ and therefore $\Re_{\ell}=\Re_{\ell+1}=\Re_{\ell+2}=\ldots$. Thus

$$
\Re=\sum_{p \geq 1} \Re_{p}=\sum_{p=1}^{n_{1}} \Re_{p}
$$

The subspace above can also be written in the form:

$$
\begin{aligned}
& \Re=\sum_{\substack{k, k^{\prime} \in\{0,1\}, k+k^{\prime}=1 \\
j_{1}, \ldots, j_{n_{1}-1} \in\left\{0,1, \ldots, n_{1}-1\right\} \\
\mathcal{A}_{1}, \ldots, \mathcal{A}_{n_{1}-1} \in\left\{A_{1}, A^{\prime}{ }_{1}\right\}}} \operatorname{Im}\left[\mathcal{A}_{1}^{j_{1}} \ldots \mathcal{A}_{n_{1}-1}^{j_{n_{1}-1}} B_{1}^{k} B^{\prime k^{\prime}}\right] \\
&
\end{aligned}
$$

We will denote by $\mathcal{R}$ the set of all reachable states and by $\mathcal{C}$ the set of all controllable states of the switching system $\Sigma$. It is clear that

$$
\mathcal{R} \subseteq\left(\Re \oplus<\mathcal{N} \mid B_{2}>\right) \cup\left(\Re \oplus<\mathcal{N}^{\prime} \mid B^{\prime}{ }_{2}>\right)
$$

and that

$$
\mathcal{C} \subseteq\left(\Re \oplus<\mathcal{N} \mid B_{2}>\right) \cup\left(\Re \oplus<\mathcal{N}^{\prime} \mid B^{\prime}{ }_{2}>\right)
$$

Our goal is to prove that the converse inclusions are also true.

In order to prove the desired characterization of the sets $\mathcal{R}$ and $\mathcal{C}$, we will need the following Lemmas.

Lemma 1: Let us assume that $x_{f}^{1} \in \Re$. Then there always exists $t_{f}>t_{0}$, a path $\bar{\theta}:\left[t_{0}, t_{f}\right] \longrightarrow M=\{1,2\}$ determined by a switching sequence $\bar{\sigma}$, and a path $\widetilde{\theta}:\left[t_{0}, t_{f}\right] \longrightarrow$ $M=\{1,2\}$ determined by a switching sequence $\widetilde{\sigma}$ such that $\Phi\left(t_{f}, t_{0}, x_{0}, u, \bar{\sigma}\right)=\Phi\left(t_{f}, t_{0}, x_{0}, u, \widetilde{\sigma}\right)=x_{f}^{1}$ and $\bar{\sigma}\left(t_{f}^{-}\right)=1$, $\tilde{\sigma}\left(t_{f}^{-}\right)=2$.

Proof: Since $x_{f}^{1} \in \Re$, then also $e^{-A_{1}} x_{f}^{1} \in \Re$. Then there exists $t_{p}>t_{0}$, a control $u$ and a switching sequence $\sigma$

$$
\left\{\left(\left[t_{0}, t_{1}\right), \sigma\left(t_{0}^{+}\right)\right), \ldots,\left(\left[t_{p}, t_{p+1}\right), \sigma\left(t_{p}^{+}\right)\right)\right\}
$$

such that $e^{-A_{1}} x_{f}^{1}=\Phi\left(t_{p}, t_{0}, 0, u, \sigma\right)$. It is enough to prove that in the case where $\sigma\left(t_{p}^{+}\right)=2$, we can construct a new switching sequence $\bar{\sigma}$,

$$
\left\{\left(\left[t_{0}, t_{1}\right), \bar{\sigma}\left(t_{0}^{+}\right)\right), \ldots,\left(\left[t_{f}, t_{f+1}\right), \bar{\sigma}\left(t_{f}^{+}\right)\right)\right\}
$$

with $\bar{\sigma}\left(t_{f}^{+}\right)=1$.

Let $t_{f}$ be $t_{p+1}$ and consider the switching sequence $\bar{\sigma}$ :

$$
\begin{aligned}
& \left\{\left(\left[t_{0}, t_{1}\right), \bar{\sigma}\left(t_{0}^{+}\right)=\sigma\left(t_{0}^{+}\right)\right), \ldots,\right. \\
& \left.\quad\left(\left[t_{p}, t_{p+1}\right), \bar{\sigma}\left(t_{p}^{+}\right)=\sigma\left(t_{p}^{+}\right)\right),\left(\left[t_{p+1}, t_{p+1}+1\right), \bar{\sigma}\left(t_{p+1}^{+}\right)=1\right)\right\}
\end{aligned}
$$

Straightforward computations show that $\Phi\left(t_{f}, t_{0}, 0, u, \bar{\sigma}\right)=$ $x_{f}^{1}$.

Lemma 2: Let us assume that $\left(x_{f}^{1}, x_{f}^{2}\right) \in \mathbb{R}^{n_{1}+n_{2}}$ is of the form $x_{f}^{1}=\Phi\left(t_{f}, t_{0}, x_{0}, u, \sigma\right)$ and $x_{f}^{2}=-\sum_{i=0}^{h-1} \mathcal{N}^{i} B_{2} \lambda_{i}$, for some $\lambda_{i} \in \mathbb{R}^{m}$. Then there always exists a $\bar{u}(t)$ such that $\Phi\left(t_{f}, t_{0}, x_{0}, \bar{u}, \sigma\right)=x_{f}^{1}$ and $-\sum_{i=0}^{h-1} \mathcal{N}^{i} B_{2} \bar{u}^{(i)}\left(t_{f}\right)=x_{f}^{2}$.

Proof: After Lemma 1, we know that we can assume that $\sigma\left(t_{f}^{-}\right)=1$.

Let us denote

$E_{0}=e^{A_{\sigma\left(t_{p}\right)}\left(t_{f}-t_{p}\right)} \cdot \ldots \cdot e^{A_{\sigma\left(t_{1}\right)}\left(t_{2}-t_{1}\right)} \cdot e^{A_{\sigma\left(t_{0}\right)}\left(t_{1}-t_{0}\right)}$

$E_{1}=e^{A_{\sigma\left(t_{p}\right)}\left(t_{f}-t_{p}\right)} \cdot \ldots \cdot e^{A_{\sigma\left(t_{1}\right)}\left(t_{2}-t_{1}\right)}$

...

$E_{p}=e^{A_{\sigma\left(t_{p}\right)}\left(t_{f}-t_{p}\right)}$

$I_{1}=\int_{t_{0}}^{t_{1}} e^{A_{\sigma\left(t_{0}\right)}\left(t_{1}-\tau\right)} B_{\sigma\left(t_{0}\right)} u(\tau) d \tau$

$I_{p}=\int_{t_{p-1}}^{t_{p}} e^{A_{\sigma\left(t_{p-1}\right)}\left(t_{p}-\tau\right)} B_{\sigma\left(t_{p-1}\right)} u(\tau) d \tau$

$I_{p+1}=\int_{t_{p}}^{t_{f}} e^{A_{\sigma\left(t_{p}\right)}\left(t_{f}-\tau\right)} B_{\sigma\left(t_{p}\right)} u(\tau) d \tau$

Then:

$$
x_{f}^{1}=E_{0} x_{0}+E_{1} I_{1}+\ldots+E_{p} I_{p}+I_{p+1}
$$

Basic properties of matrix functions allow to prove that $I_{p+1} \in<A_{1} \mid B_{1}>$. Let us call now $I_{p+1}^{\prime}=$ $\int_{t_{p}}^{t_{f}} e^{A_{\sigma\left(t_{p}\right)}\left(t_{f}-\tau\right)} B_{\sigma\left(t_{p}\right)} U(\tau) d \tau$, where $U(\tau)$ denotes the control used defined on $\left[t_{p}, t_{f}\right]$ by Dai in [4], proof of Theorem $2-1$, pp. 25-27. It is not difficult to prove that $I_{p+1}=I^{\prime}{ }_{p+1}$, therefore if we consider the piece-wise continuous control $\bar{u}(t)$ defined by: $\bar{u}(t)=u(t)$ if $t<t_{p}$ and $\bar{u}(t)=U(t)$ if $t \in\left[t_{p}, t_{f}\right], x_{f}^{1}=\Phi\left(t_{f}, t_{0}, x_{0}, \bar{u}, \sigma\right)$ and moreover we have that $x_{f}^{2}=-\sum_{i=0}^{h-1} \mathcal{N}^{i} B_{2} \bar{u}^{(i)}\left(t_{f}\right)$. This completes the proof.

Now we can state the main Theorems.

Theorem 1: The set of reachable states for system $\Sigma$ is:

$$
\mathcal{R}=\left(\Re \oplus<\mathcal{N} \mid B_{2}>\right) \cup\left(\Re \oplus<\mathcal{N}^{\prime} \mid B^{\prime}{ }_{2}>\right)
$$

Proof: Let us consider an element $x=\left(x_{1}, x_{2}\right) \in \Re \oplus<$ $\mathcal{N} \mid B_{2}>$. There exists (see [12]) $t_{f}>t_{0}$, a switching sequence $\sigma$ and a control $u(t)$ such that $x_{1}=\Phi\left(t_{f}, t_{0}, x_{0}, u, \sigma\right)$. Assuming that $\sigma\left(t_{f}^{-}\right)=1$, after Lemma 2, we also know that there exists a control $\bar{u}(t)$ such that $x_{1}=\Phi\left(t_{f}, t_{0}, 0, \bar{u}, \sigma\right)$ and $x_{2}=-\sum_{i=0}^{h-1} \mathcal{N}^{i} B_{2} \bar{u}^{(i)}\left(t_{f}\right)$. Therefore $\left(x_{1}, x_{2}\right)$ is a reachable state for $\stackrel{i=0}{\Sigma}$.

In the case where $\sigma\left(t_{f}^{-}\right)=2$, we know, after Lemma 1 , that there exists another switching sequence $\bar{\sigma}$, $\left\{\left(\left[t_{0}, t_{1}\right), \sigma\left(t_{0}^{+}\right)\right), \ldots,\left(\left[t_{\ell}, t_{\ell+1}\right), \sigma\left(t_{\ell}^{+}\right)\right),\left(\left[t_{\ell+1}, t_{\ell+1}+1\right), 1\right)\right\}$. Being now in a situation exact to that above, we can proceed in an analogous way.

The case where $x=\left(x_{1}, x_{2}\right) \in \Re \oplus<\mathcal{N}^{\prime} \mid B^{\prime}{ }_{2}>$ can be handled similarly.

Using a similar reasoning, we obtain the following characterization of the set of controllable states. Note that the set of such states is the same as the set of reachable states.

Theorem 2: The set of controllable states for system $\Sigma$ is:

$$
\mathcal{C}=\left(\Re \oplus<\mathcal{N} \mid B_{2}>\right) \cup\left(\Re \oplus<\mathcal{N}^{\prime} \mid B^{\prime}{ }_{2}\right)>
$$

Controllability and reachability of system $\Sigma$ may be deduced from Theorems above. First, we recall the usual definition of controllable and reachable systems. 
Definition 6: System $\Sigma$ is said to be reachable if every state $x \in \mathbb{R}^{n}$ is reachable.

Definition 7: System $\Sigma$ is said to be controllable if every state $x \in \mathbb{R}^{n}$ is controllable.

As a consequence of the Theorems above, controllable and reachable switched singular systems may be characterized as follows.

Corollary 1: For system $\Sigma$, the following conditions are equivalent:

(a) $\Sigma$ is controllable.

(b) $\Sigma$ is reachable.

(c) $\mathbb{R}^{n}=\Re \oplus<\mathcal{N} \mid B_{2}>$ or $\mathbb{R}^{n}=\Re \oplus<\mathcal{N}^{\prime} \mid B^{\prime}{ }_{2}>$.

Thus, $\Sigma$ is controllable (reachable) if for any states $x_{0}$, $x_{f}$, there exists $t_{f}>t_{0}$, a switching path $\theta$ determined by a switching sequence $\sigma$ and a control $u$ such that $\Phi\left(t_{f}, t_{0}, x_{0}, u, \sigma\right)=x_{f}$.

\section{CONCLUSiON}

We have determined the space of controllable (and reachable) states and characterized controllable (reachable) switched singular systems satisfying the "equisingularity condition". Note that these results can be easily generalized to the case of a switched system composed of a family which consists of more than two linear singular systems.

Observability conditions and the set of observable and reconstructible states can be deduced of the results above by the principle of duality. Observability (respectively, reconstructibility) of a switched singular system is equivalent to reachability (respectively, controllability) of its dual system.

In the example of $\S 3$, the set of reachable states and the set of controllable states is $\operatorname{Im}\left[(1,0,0)^{t},(0,1,0)^{t}\right]$. That is to say, every value for $I(t)$ and $e_{C}(t)$ are possible. Obviously, a value $x_{3} \neq 0$ can not be achieved (note that $x_{3}(t)=0$ for all $t$ according to Ohm's law).

\section{REFERENCES}

[1] R.W. Brockett, J.R. Wood, Electrical networks containing controlled switches, Applications of Lie Groups Theory to Nonlinear Networks Problems, Supplement to IEEE Inter. Symp. Circuit, 1974.

[2] S. L. Campbell, N. J. Rose, A second-order singular linear system arising in electric power systems analysis, Int. J. Systems Sci. 13 (1), 1982.

[3] D. Corona, A. Giua, C. Seatzu, Optimal control of hybrid automata: design of a semiactive suspension, Contr. Engin. Practice 12 (10), 2004.

[4] L. Dai, Singular Control Systems, New York, U.S.: Springer, 1989.

[5] A. Feuer, G. C. Goodwin, M. Salgado, Potential benefits of hybrid control for linear time invariant plants, American Contr. Conf., 1997.

[6] S. S. Ge, C. C. Hang, T. H. Lee, T. Zhang, Stable Adaptive Neural Network Control, Boston: Kluwer, 2001.

[7] B. C. Haggman, P. R. Bryant, Solution of Singular Constrained Differential Equations: A Generalization of Circuits Containing Capacitor-Only Loops and Inductor-Only Cutsets, IEEE Trans. Circuits and Systems, CAS-31 (12), 1984.

[8] W.L. De Koning, Digital optimal reduced-order control of pulse-widthmodulated switched linear systems, Automatica 39 (11), 2003.

[9] D. G. Luenberger, Dynamic equation in descriptor form, IEEE Trans. Aut. Control, AC-22, 1977.

[10] L. R. Petzold, Differential/Algebraic Equations are not ODE's, SIAM J. Sci. Stat. Comp. 3, 1982.

[11] S.P. Singh, R.W. Liu, Existence of state equation representation of linear large-scale dynamic systems, IEEE Trans. Circuit Theory, CT-20 (5), 1973.
[12] Z. Sun, S. S. Ge, Switched Linear Systems, London, England: Springer, 2005.

[13] C. Wang, L. Dai, Singular dynamic systems - A survey, Control Theory and Appl. 1, 1986. 